\title{
micromachines
}

ISSN 2072-666X

www.mdpi.com/journal/micromachines

Article

\section{Multi-Input Multi-Output Integrated Ionic Polymer-Metal Composite for Energy Controls}

\author{
Gou Nishida ${ }^{1, *}$, Motonobu Sugiura ${ }^{2}$, Masaki Yamakita ${ }^{2}$, Bernhard Maschke ${ }^{3}$ and \\ Ryojun Ikeura ${ }^{1,4}$
}

1 RIKEN-TRI Collaboration Center for Human-Interactive Robot Research, Advanced Science Institute, RIKEN, 2271-130 Anagahora, Shimoshidami, Moriyama-ku, Nagoya, Aichi, 463-0003, Japan

2 Department of Mechanical and Control Engineering, Tokyo Institute of Technology, 2-12-1 Oookayama, Meguro, Tokyo, 152-8552, Japan; E-Mails: openthenext6621@gmail.com (M.S.); yamakita@ac.ctrl.titech.ac.jp (M.Y.)

3 Université de Lyon, Lyon, F-69003, France; Université Lyon 1, Faculté Sciences et Technologie, France; CNRS, UMR5007, Laboratoire d'Automatique et Génie des Procédés, Villeurbanne, F-69622, France; E-Mail: maschke@lagep.univ-lyon1.fr

4 Department of Mechanical Engineering, Mie University, Kamihama 1515, Tsu, Mie, 514-8507, Japan; E-Mail: ikeura@ss.mach.mie-u.ac.jp

* Author to whom correspondence should be addressed; E-Mail: g.nishida@ieee.org; Tel.: +81-52-736-5870; Fax: +81-52-736-5871.

Received: 22 December 2011; in revised form: 13 February 2012 / Accepted: 15 February 2012 / Published: 29 February 2012

\begin{abstract}
This paper presents an integrated sensor/actuator device with multi-input and multi-output designed on the basis of a standard control representation called a distributed port-Hamiltonian system. The device is made from soft material called an ionic polymer-metal composite (IPMC). The IPMC consists of a base film of a polyelectrolyte gel and a double layer of plated metal electrodes. The electrodes of the experimental IPMC are sectioned, and it is implemented as a control system with four pairs of inputs/outputs. We stabilize the system, and detect changes in dynamics by using the control representation.
\end{abstract}

Keywords: IPMC; polymer; integrated actuators/sensors; controls of PDEs 


\section{Introduction}

Electro-active polymers (EAPs) are deformed by an electric stimulus. One of these EAPs, called an ionic polymer metal composite (IPMC, Figure 1) [1,2], is used as an integrated device composed of sensors and actuators. IPMC is a film of a polyelectrolyte gel sandwiched between two layers of metal (gold) electrodes plated by a chemical process. IPMC can be bent by applying a low voltage (1-2 V) between the electrodes at 10-100 Hz [1-4], and its deformation depends on the thickness of the film and the type of counter ions in the gel, e.g., sodium ions $\mathrm{Na}^{+}$or tetraethyl-ammonium ions $\mathrm{TEA}^{+}[5,6]$. IPMC is flexible, chemically stabile, noiseless, durable (it is possible to bend it over $1 \times 10^{6}$ times [1,7]), and lightweight. Thus, IPMC is expected to be used in sensor/actuator devices with back-drivability for a living environment, i.e., it is soft to the touch.

Figure 1. Ionic polymer metal composite (IPMC).

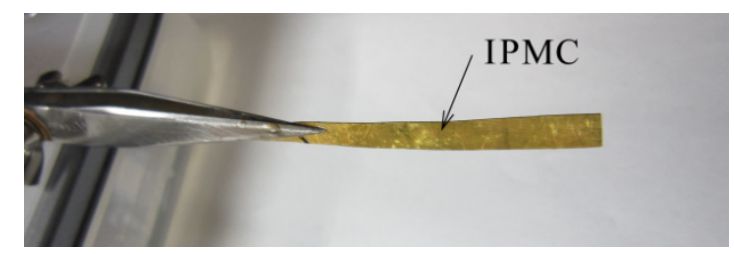

IPMC consists of three physical systems, i.e., the electrical system modeling the characteristic of the electrodes, the mechanical system as a flexible beam, and their electro-mechanical coupling system explained in the following. Cations (e.g., $\mathrm{Na}^{+}$or $\mathrm{TEA}^{+}$) and water molecules in the gel are transferred to the side of the negative electrode by the potential between the electrodes. This side of the gel is swollen by the transfer. As a result, the swelling mechanically bends the whole film (Figure 2). This transfer process can be modeled by coupling the water transport generated by an electric field, called electro-osmosis, and the electric field generated by water transport, called streaming potential, in the polymer gel on a spatial scale of $100 \mu \mathrm{m}$, and a time scale of about $1 \mathrm{~s}\left(\mathrm{Na}^{+}\right)-100 \mathrm{~s}\left(\mathrm{TEA}^{+}\right)$[8]. The mechanical motion of IPMC can be described as a flexible beam [9] on a spatial scale of $10 \mathrm{~cm}$ and a time scale of about $0.03 \mathrm{~s}$. Hence, analytical models of IPMC are nonlinear systems of partial differential equations (PDEs).

Figure 2. Mechanism for IPMC.

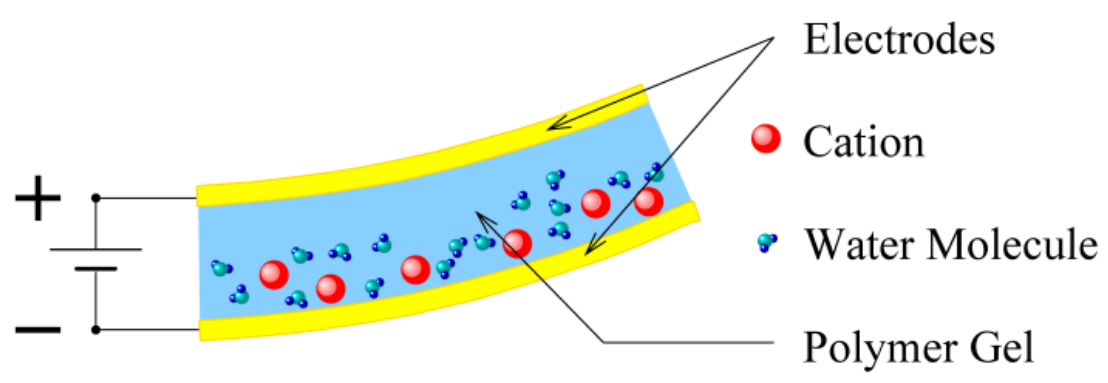

This paper presents an experimental example of a micro-mechanical construction incorporating controls. Micro-devices used in sub-centimeter environments are affected by the viscosity of the medium; therefore, they are subject to nonlinearities and must be treated as distributed parameter systems when control models are constructed for them. However, the design of such controls generally 
involves nonlinear systems and PDEs. An ordinary PDE control, for instance, assigns a state to the system domain. Thus, this method is based on analytical solutions to the systems.

On the other hand, distributed port-Hamiltonian (DPH) systems [10] are a standard control representation using nonlinear PDEs for energy controls, called passivity-based controls [11]. Passivity-based controls create energy flows in order to vary energy levels or the global minimum state to stabilize the system. DPH systems are based on the energies of the systems, that is to say, their geometry, because they are described in the language of differential geometry, e.g., they invoke Stokes theorem. They are not so good for control designs depending on analytical solutions, e.g., ones based on control performances; however, they can be applied to wide classes of complicated systems, such as IPMC, that are difficult to treat analytically.

Moreover, DPH systems satisfy a power balance equation between the change in energy distributed throughout the system domain inside a boundary and the energy on the boundary. We can use this power balance for passivity-based boundary controls. This paper presents two experimental examples, i.e., stabilizing IPMC in terms of boundary control inputs and detecting dynamical changes in IPMC in terms of boundary control outputs. These boundary control inputs/outputs are systematically derived from the DPH system representation.

\section{Experimental System and Control Model}

This section is devoted to explaining the experimental IPMC system and its control model.

\subsection{Construction of Sectioned IPMC and Experimental System}

IPMC acts as an actuator when we apply a voltage to electrodes and as a sensor when we measure the counteraction voltage between electrodes without the voltage input. The sensor mode has been studied in, e.g., [12-20]. The experimental IPMC was implemented as a control system with four distributed inputs and four distributed outputs. The pattern of inputs/outputs for the electrode was made by laser cutting (Figure 3).

Figure 3. Sectioned IPMC with Multiple Inputs and Multiple Outputs.

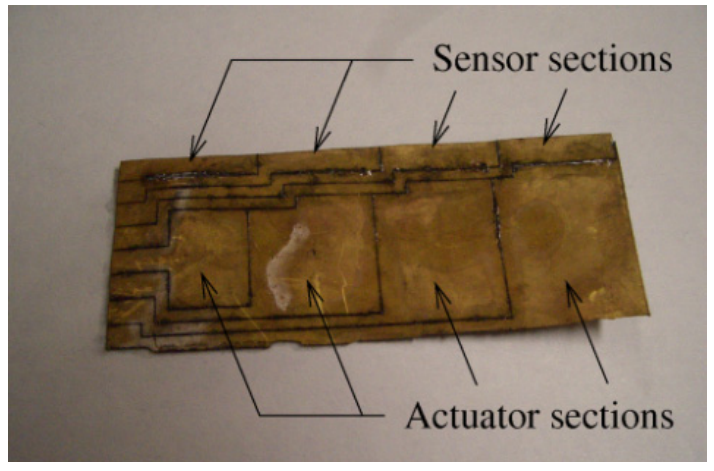

The spatial dimensions of the IPMC are $51 \mathrm{~mm}$ (length) $\times 19.2 \mathrm{~mm}$ (width) $\times 0.18 \mathrm{~mm}$ (thickness) (Figure 4). The sectioned electrodes consist of four actuator areas and four sensor areas, and they are connected to one side of the IPMC that is used as an interface connector to the control and measurement system. The control and measurement system is composed of a personal computer, an 
A/D convertor, a D/A convertor, amplifiers, and a laser measurement device (Figure 5). The inputs and outputs are processed by the personal computer. The signals for the inputs/outputs are transmitted through the convertors and the amplifiers. The interface side of the IPMC is fixed at $x^{0}$ in Figure 4. We positioned the IPMC horizontally with respect to the surface of the ground. We measured the share displacement $w^{i}\left(x^{i}, t\right)$ vertically at each position $x^{i}$ for $1 \leq i \leq 4$ with a laser measurement device to verify the sensor output of the IPMC. We assumed that the spatial difference in velocity $w_{t}^{i}-w_{t}^{i-1}$ for $1 \leq i \leq 4$ would be in proportion to the output voltage $V_{i}$ of the $i$-th sensor from the left, i.e., $V_{i} \propto C\left(w_{t}^{i}-w_{t}^{i-1}\right)$, where $C$ is a constant and $w_{t}^{0}=0$. Accordingly, we can approximate the spatial partial derivatives of the share displacements from the spatial differences.

Figure 4. Functions of Sectioned IPMC.

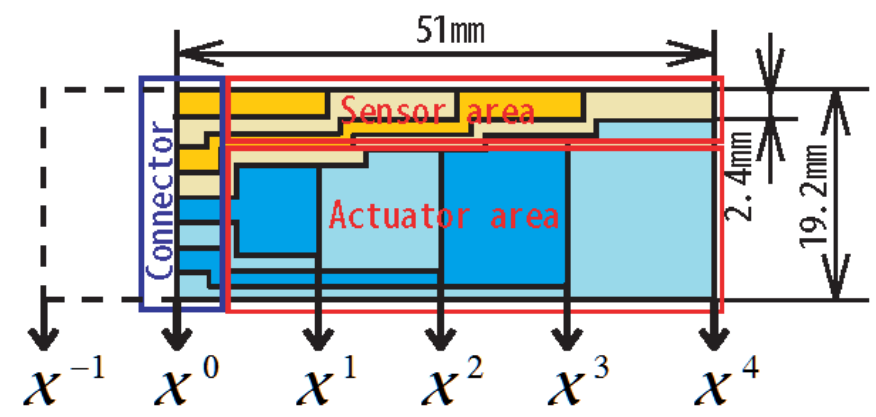

Figure 5. Experimental System.

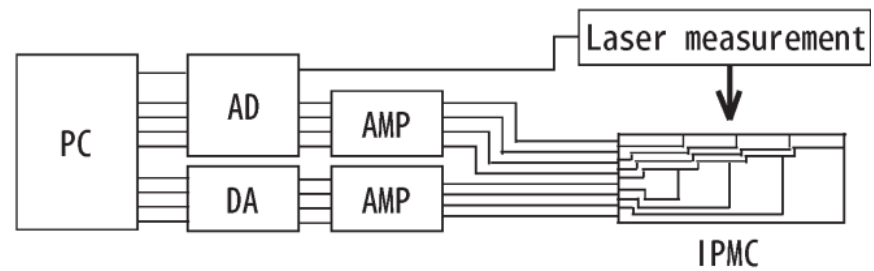

\subsection{Control Model of IPMC}

Flexible structures are modeled in terms of PDEs. The mechanical characteristics of IPMC can be modeled, e.g., by using the Euler-Bernoulli beam model, the Timoshenko beam model, and the model with large deformations [21]. These beam models can be expressed as DPH systems [9]. The overall motion of IPMC is nonlinear; therefore, the large deformation beam would the best choice for the motion analysis. However, the large deformation beam is expressed by three physical variables, i.e., the axial displacement $y$, shear displacement $w$, and rotation $\theta$, and we cannot directly obtain the output with respect to the rotation from the experimental IPMC. Thus, we decided to employ the Euler-Bernoulli beam model instead as a real-time control model. The Euler-Bernoulli beam model is actually a reduced large deformation beam made by assuming $w_{x}=\theta$ and making a simplification [9], wherein the subscript $x$ means the derivative with respect to the spatial coordinate $x$. However, we must measure (higher order) spatial derivatives of $w$ instead of $\theta$.

The Euler-Bernoulli beam model includes up to fourth-order spatial derivatives. Such higher-order PDEs are modeled in terms of higher-order DPH systems [22]. In the case of IPMC, the DPH system is only second order. The higher-order DPH systems have non-trivial higher-order boundary ports that 
are derived from the integration by parts formula (see Appendices A.1 and A.2). These special inputs/outputs pairs never appear in the standard (first order) DPH systems; therefore, they offer the possibility of applying passivity-based controls to IPMC. We verified this possibility by means of the experimentation described in Section 3.

On the other hand, film structures are modeled in terms of two-dimensional PDEs, i.e., flexible plate equations. The film model is used for, e.g., controlling the shape of the film. However, one-dimensional PDEs are appropriate control models in the case of the experimental IPMC, because, the control degree of the IPMC is unidirectional regardless of the dimension. Hence, we chose the DPH representation having a one dimensional Euler-Bernoulli beam equation as the control model.

Let us consider the one-dimensional Euler-Bernoulli beam model on the interval $[0, L]$ :

$$
A_{\rho} w_{t t}+E I w_{x x x x}=0
$$

where $L$ is the length of the beam, $A_{\rho}$ is the mass per unit length, $E I$ is the flexural stiffness, $w=w(t, x)$ is the share displacement, $t$ is the time coordinate, and $x$ is the spatial coordinate. Here, the subscript of $w$ means partial derivatives with respect to $t$ or $x$. Equation (1) can be transformed into a second order DPH system:

$$
\left[\begin{array}{l}
f_{p} \\
f_{q}
\end{array}\right]=\left[\begin{array}{cc}
0 & -\frac{\partial^{2}}{\partial x^{2}} \\
\frac{\partial^{2}}{\partial x^{2}} & 0
\end{array}\right]\left[\begin{array}{l}
e_{p} \\
e_{q}
\end{array}\right]
$$

where we have defined the variables:

$$
\left[\begin{array}{l}
f_{p} \\
f_{q}
\end{array}\right]=\left[\begin{array}{l}
-\frac{\partial}{\partial t} A_{\rho} w_{t} \\
\frac{\partial}{\partial t} w_{x x}
\end{array}\right], \quad\left[\begin{array}{l}
e_{p} \\
e_{q}
\end{array}\right]=\left[\begin{array}{c}
w_{t} \\
-E I w_{x x}
\end{array}\right]
$$

Note that the first row in Equation (2) is equivalent to that in Equation (1), and the second row in Equation (2) is an identity [7]. The advantage of this system representation is that it always satisfies the power balance equation at an instant in time:

$$
\int_{0}^{L}\left(e_{p} f_{p}+e_{q} f_{q}\right) d x+\left(e_{1}^{L} f_{1}^{L}+e_{2}^{L} f_{2}^{L}\right)-\left(e_{1}^{0} f_{1}^{0}+e_{2}^{0} f_{2}^{0}\right)=0
$$

where we have defined the variables at the boundaries $x=0, L$,

$$
\begin{gathered}
{\left[\begin{array}{l}
f_{1}^{0} \\
e_{1}^{0}
\end{array}\right]=\left[\begin{array}{c}
\left.w_{t x}\right|_{x=0} \\
\left.E I w_{x x}\right|_{x=0}
\end{array}\right],\left[\begin{array}{l}
f_{2}^{0} \\
e_{2}^{0}
\end{array}\right]=\left[\begin{array}{c}
\left.w_{t}\right|_{x=0} \\
-\left.E I w_{x x x}\right|_{x=0}
\end{array}\right] \text { and }} \\
{\left[\begin{array}{l}
f_{1}^{L} \\
e_{1}^{L}
\end{array}\right]=\left[\begin{array}{c}
\left.w_{t x}\right|_{x=L} \\
\left.E I w_{x x}\right|_{x=L}
\end{array}\right],\left[\begin{array}{l}
f_{2}^{L} \\
e_{2}^{L}
\end{array}\right]=\left[\begin{array}{c}
\left.w_{t}\right|_{x=L} \\
-\left.E I w_{x x x}\right|_{x=L}
\end{array}\right]}
\end{gathered}
$$

The first term in Equation (4) is equivalent to the time variation of the Hamiltonian (with a minus sign), i.e., the total energy change in the system (see Appendix A.1), and the second and third terms in Equation (4) mean energies flowing across the boundaries, which are derived in terms of Stokes theorem and integration by parts with respect to the first term in Equation (4) (see Appendix A.2). Thus, we can detect the total energy change by calculating the boundary energy flows instead of 
integrating the first term in Equation (4) over the domain. This is the basic strategy of passivity-based boundary controls of DPH systems.

\section{Control Methods and Experimental Results}

We stabilize the IPMC and detect changes in its dynamics in terms of its power balance.

\subsection{Control Method I: Stabilization}

The pair $\left(f_{j}^{i}, e_{j}^{i}\right)$ for $i=0, L$ and $j=1,2$ in Equations (5) and (6) means a collocated pair of boundary inputs and outputs for passivity-based controls. Let us consider the pair $\left(f_{2}^{0}, e_{2}^{0}\right)$ in Equation (5) for the IPMC. The third-order derivative of the share displacement $w_{x x x}^{0.5}$ at $x^{0.5}=L / 8$ can be approximated as $w_{x x x}^{0.5} \cong\left\{\left(w^{2}-w^{1}\right)-\left(w^{1}-w^{0}\right)\right\}-\left\{\left(w^{1}-w^{0}\right)-\left(w^{0}-w^{-1}\right)\right\}=w^{2}-3 w^{1}$, where $w^{-1}$ is the share displacement at virtual position $x^{-1}$ in Figure 4 . We regard $-E I w_{x x x}^{0.5}$ as the output $e_{2}^{0}$ in Equation (5). Hence, we send the feedback input $f_{2}^{0}=-K e_{2}^{0}=K E I\left(w^{2}-3 w^{1}\right)$ to the first actuator distributed on the interval $\left[x^{0}, x^{1}\right]$, where the input voltage of the actuator is determined by $V_{1}=C w_{t}^{1}$ for a constant $C$, and $K$ is feedback gain. We applied a band-pass filter for $0.1 \leq f \leq 1.0 \mathrm{~Hz}$ to the output voltages, because the above assumption is valid around that frequency range.

The input voltage to the first actuator and the sharer displacements $w^{1}$ and $w^{2}$ are shown in Figure 6 . The control input $f_{2}^{0}$ is added to the first actuator after $t=5.0$. Two impact disturbances are applied to the tip of the IPMC at $t=1.8$ and $t=6.4$. We can see that the residual vibration in the controlled IPMC after $t=5.0$ decreases more rapidly than in the uncontrolled IPMC before this time. This means the total energy of the first term in Equation (4) is dissipated through the second and third terms in Equation (4), because the negative feedback applied to boundary variables $f_{2}^{0}$ and $e_{2}^{0}$ acts as a dissipative element.

Figure 6. Experimental Results for Stabilization.
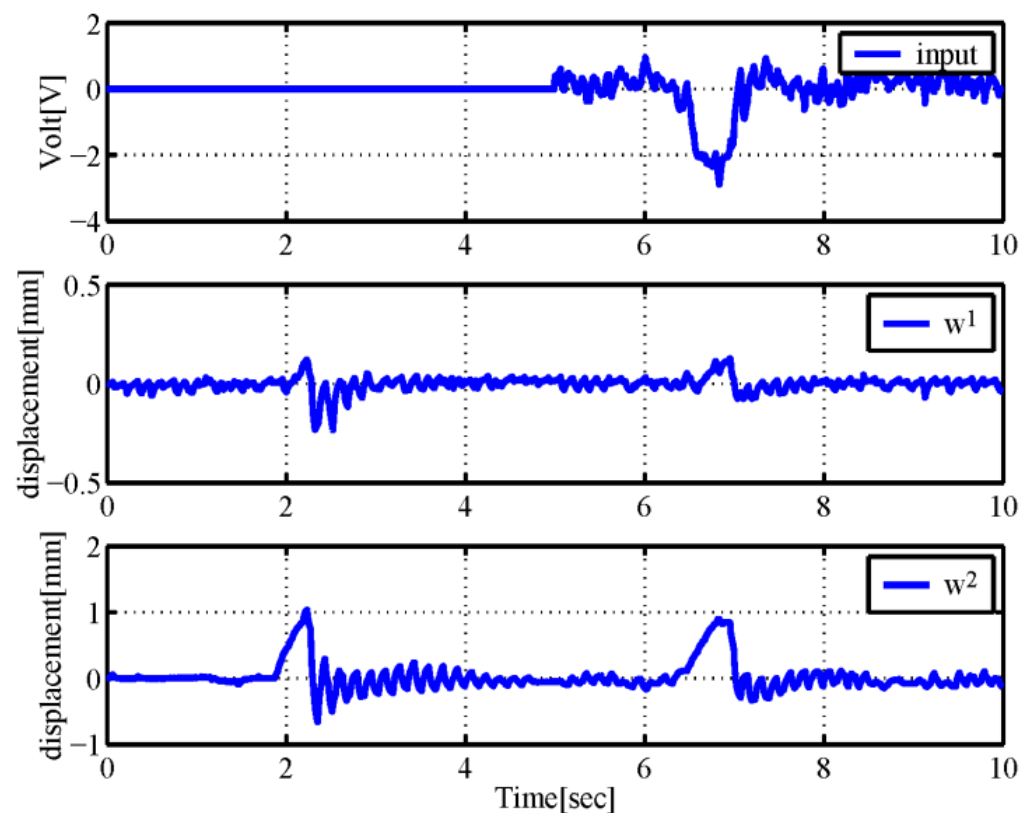


\subsection{Control Method II: Detection of Dynamical Changes}

This section explains how we detect the change in the dynamics of the IPMC in terms of the power balance Equation (4). Time-independent Hamiltonian systems are conservative, i.e., time invariant with respect to energy. However, actual systems have dissipative elements, e.g., viscosity damping or distributed registers. Indeed, the swelling of IPMC relaxes by dissipation, but this effect has not been modeled in Equation (1). If the total energy stored in the system is dissipated inside the system domain, the observed boundary energy flow calculated with the second and third term in Equation (4) will be less than the total energy. Moreover, the change in the dissipation rate can be detected from the difference in observed energies.

We conducted experimental tests in two dissipative situations. The first situation was where one side of the overall motion of the IPMC was blocked around $x^{3}$ by placing an external object after $t=14.0$. The second situation was where the tip of the IPMC was soaked in water and the water level was raised after $t=15.0$. We believed these environmental changes might increase the energy dissipated by the IPMC.

Figures 7 and 8 plot the responses of the IPMC to a sinusoidal input of $3 \mathrm{~Hz}$. The responses were detected by the laser measurement device placed at $x^{1}$ (laser output), and the total boundary energy flows in the first and second sensor areas (total pow1 and total pow2) were calculated from the time integral of the product of input $f_{2}^{0}$ and output $e_{2}^{0}$ in Equation (5). The ranges of the figures have been normalized to be dimensionless. The estimated responses before the time of the change in dissipations are also plotted (estimates).

Figure 7 indicates that dissipation increased when the object came into contact after $t=14.0$, and the change in the dissipation rate can be seen as a change in the slopes of the second and third graphs. We can see from Figure 8 that a change in dissipation is detected, because dissipation constantly increases because of the viscous drag of the water after $t=15.0$.

Figure 7. Experimental Results on Detecting Dynamical Changes (obstacle).
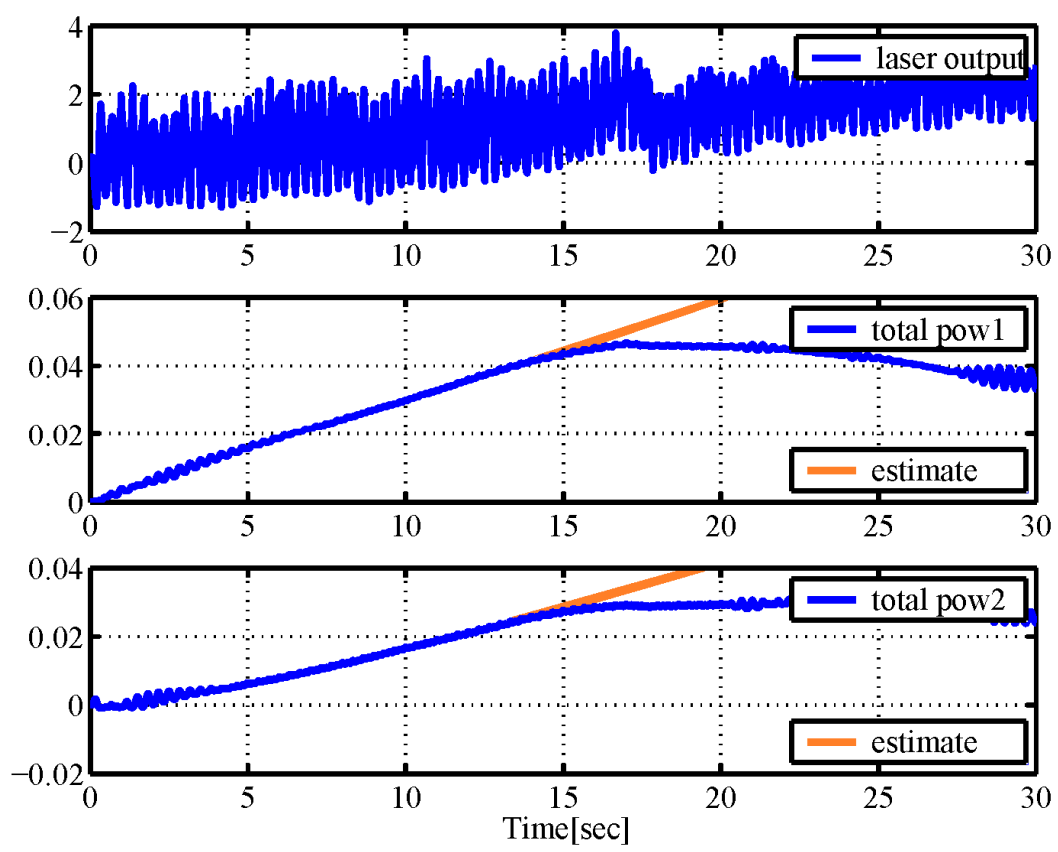
Figure 8. Experimental Results on Detecting Dynamical Changes (water).
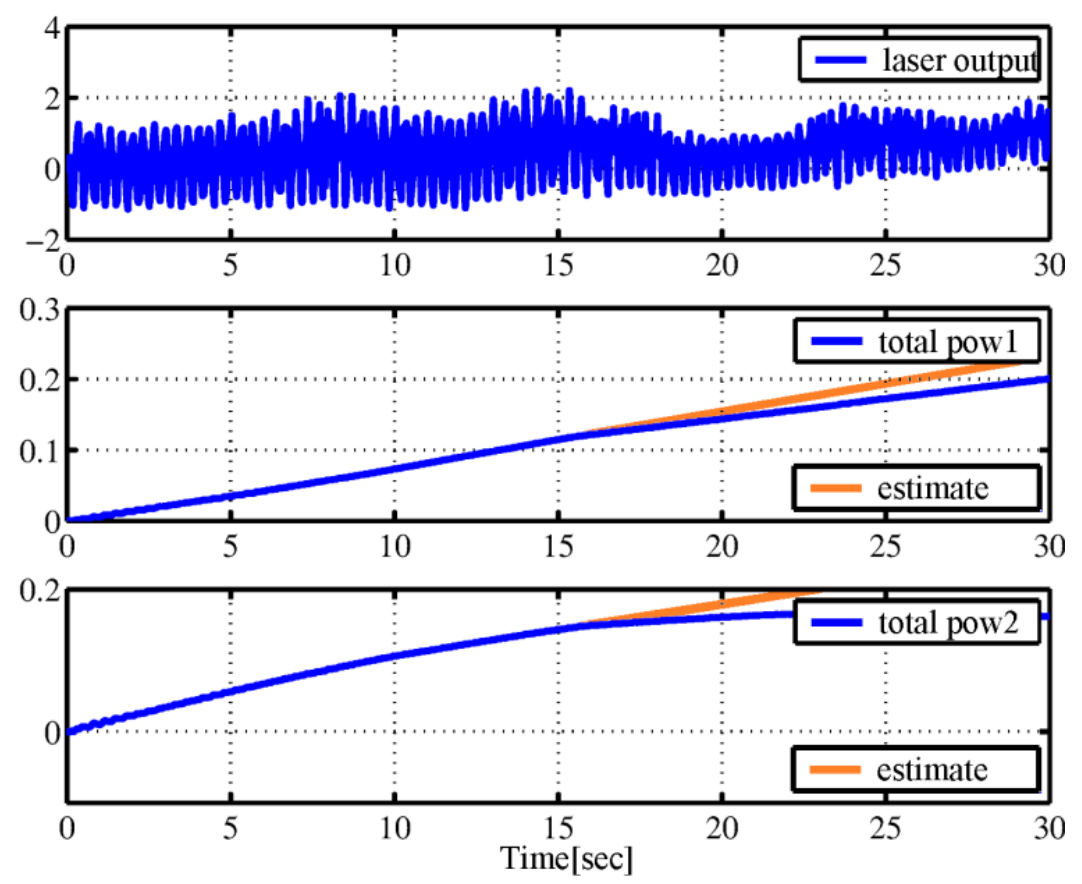

It is difficult to detect the above changes solely from the laser outputs, which are the closest data to the real motion of the IPMC, because the amplitudes of the laser outputs are not so stable. We guess that the IPMC flexibly bends because of the obstruction. However, we can clearly detect the changes from the power balance from the change in the slopes calculated from the control model.

The amount of energy dissipated in Figure 7, i.e., the difference in the slopes of the total measured power and the estimate, is larger than in Figure 8. The movement of the IPMC is completely blocked by the obstacle in the first situation. However, the viscous drag of the water in the second situation does not stop it from moving. Therefore, we can guess that the dissipated energy is much larger in the first situation than in the second situation.

\section{Conclusions}

This paper presented experimental results on a multi-input/multi-output integrated ionic polymer metal composite (MIMO-IPMC) controlled by the passivity-based controls having a distributed port-Hamiltonian (DPH) system representation. The sectioned electrodes in the experimental IPMC had four inputs and four outputs. The IPMC was modeled with an Euler-Bernoulli equation, and the model was transformed into a DPH system with four pairs of collocated inputs and outputs for passivity-based controls. We applied two passivity-based controls to the experimental IPMC, i.e., we stabilized the system and detected the change in the dissipative structure in terms of the power balance equation of the DPH system. These experimental results illustrated the effectiveness of the control methods for MIMO-IPMC.

The DPH system representation can be applied to multi-physical and multi-scale systems including dissipative elements [9]. That is, particular pairs of control inputs and outputs for passivity-based controls are systematically derived from complicated system models. We expect this formalism will be used for various micro-machines. The continuous system representation can be extended to a 
discretized system representation for numerical calculations [25]. We are interested in the applications of unified methods of numerical analyses and automatic controls based on DPH systems to micro-machines that require precise mechanical designs.

\section{Acknowledgments}

This work was supported by Grants-in-Aid for Young Scientists (B) (Nos. 19760298 and 19760169), and Scientific Research (C) (No. 19560435) from the Ministry of Education, Culture, Sports, Science and Technology and by the Japan-France Integrated Action Program (SAKURA) (No. 15994PF) of the JSPS and a Grant-in-Aid from the French Ministry of Foreign Affairs.

The authors would like to thank Kazuhiro Tanaka for his editing assistance.

\section{References}

1. Electroactive Polymer (EAP) Actuators as Artificial Muscles: Reality, Potential, and Challenges, 2nd ed.; Cohen, Y. B., Ed.; SPIE Press: Washington, DC, USA, 2004.

2. Shahinpoor, M.; Kim, K.J. Ionic polymer-metal composites: I. Fundamentals. Smart Mater. Struct. 2001, 10, 819-833.

3. Kothera, C.; Leo, D. Bandwidth characterization in themicropositioning of ionic polymer actuators. J. Intell. Mater. Syst. Struct. 2005, 16, 3-13.

4. Mallavarapu, K.; Leo, D. Feedback control of the bending response of ionic polymer actuators. J. Intell. Mater. Syst. Struct. 2001, 12, 143-155.

5. Onishi, K.; Sewa, S.; Asaka, K.; Fujiwara, N.; Oguro, K. The effects of counter ions on characterization and performance of a solid polymer electrolyte actuator. Electrochim. Acta 2001, 46, 1233-1241.

6. Yamaue, T.; Mukai, H.; Asaka, K.; Doi, M. Electrostress diffusion coupling model for polyelectrolyte gels. Macromolecules 2005, 38, 1349-1356.

7. Bennett, M.; Leo, D. Ionic liquids as stable solvents for ionic polymer transducers. Sens. Actuat. A 2004, 115, 79-90.

8. Asaka, K.; Oguro, K. Bending of polyelectrolyte membrane platinum composites by electric stimuli part II. Response kinetics. J. Electroanal. Chem. 2000, 186-198.

9. Nishida, G.; Takagi, K.; Maschke, B.; Osada, T. Multi-scale distributed parameter modeling of ionic polymer-metal composite soft actuator. Control Eng. Pract. 2011, 19, 321-334.

10. Van der Schaft, A.J.; Maschke, B.M. Hamiltonian formulation of distributed-parameter systems with boundary energy flow. J. Geom. Phys. 2002, 42, 166-194.

11. Van der Schaft, A.J. $L_{2}$-Gain and Passivity Techniques in Nonlinear Control, 2nd revised and enlarged ed.; Springer Communications and Control Engineering Series; Springer-Verlag: London, UK, 2000.

12. Chen, Z.; Tan, X.; Will, A.; Ziel, C. A dynamic model for ionic polymer-metal composite sensors. Smart Mater. Struct. 2007, 16, 1477-1488.

13. Kim, K.J.; Tadokoro, S. Electroactive Polymers for Robotic Application: Artificial Muscles and Sensors; Springer: Berlin, Germany, 2007. 
14. Tiwari, R.; Kim, K.J. Improved IPMC sensing by use of cation \& through induced nanoto-micro scale surface cracks. Proc. SPIE 2008, 6932, 69323H.

15. Nemat-Nasser, S.; Li, J. Electromechanical response of ionic polymermetal composites. J. Appl. Phys. 2000, 87, 3321-3331.

16. Kamamichi, N.; Yamakita, M.; Asaka, K. A Snake-Like Swimming Robot Using IPMC Actuator/Sensor. In Proceedings of 2006 IEEE International Conference on Robotics and Automation, Orlando, FL, USA, 15-19 May 2006; pp. 1812-1817.

17. Yamakita, M.; Sera, A.; Kamamichi, N.; Asaka, K. Integrated design of IPMC actuator/sensor. Adv. Robot. 2008, 22, 913-928.

18. Takagi, K.; Kamamichi, N.; Stoimenov, B.; Mukai, T.; Asaka, K.; Luo, Z.-W. Frequency response characteristics of IPMC sensors with current/voltage measurements. Proc. SPIE 2008, 6927, 692724.

19. Shahinpoor, M.; Henderson, B.K.; Leo, D.J. Sensing capabilities of ionic polymer-metal composites. Proc. SPIE 2001, 4328, 267-274.

20. Punning, A.; Kruusmaa, M.; Aabloo, A. Surface resistance experiments with IPMC sensors and actuators. Sens. Actuat. A: Phys. 2007, 133, 200-209.

21. Simo, J.C.; Vu-Quoc, L. On the dynamics of flexible beams under large overall motions-the plane case: Part I. J. Appl. Mech. 1986, 53, 849-854.

22. Nishida, G.; Yamakita, M. A Higher Order Stokes-Dirac Structure for Distributed-Parameter Port-Hamiltonian Systems. In Proceedings of 2004 American Control Conference, Boston, MA, USA, 30 June-2 July 2004; Volume 6, pp. 5004-5009.

23. Gotay, M.J. A multisymplectic framework for classical field theory and the calculus of variations II: Space + time decomposition. Differ. Geom. Appl. 1991, 1, 375-390.

24. Nishida, G.; Sugiura, M.; Yamakita, M.; Maschke, B.; Ikeura, R. Boundary Detection of Variational Symmetry Breaking Using Port-Representation of Conservation Laws. In Proceedings of the 48th IEEE Conference on Decision and Control, Shanghai, China, 15-18 December 2009; pp. 2861-2868.

25. Nishida, G.; Maschke, B.; Ikeura, R. Discretized Hamiltonian Systems with Distributed Energy Flows on Divisible Meshes. In Proceedings of IFAC World Congress, Milano, Italy, 28 August-2 September 2011.

\section{Appendixes}

\section{A.1. Time Variational Derivative of Hamiltonians}

Let us consider the Hamiltonian $\mathcal{H}$ of the Euler-Bernoulli beam equation:

$$
\mathcal{H}=\int_{0}^{L}\left\{\frac{\partial \mathcal{L}}{\partial w_{t}} w_{t}-\mathcal{L}\right\} d x, \quad \mathcal{L}=\int_{0}^{L} \frac{1}{2}\left\{A_{\rho}\left(w_{t}\right)^{2}-E I\left(w_{x x}\right)^{2}\right\} d x,
$$

where $\mathcal{L}$ is the Lagrangian of the equation, the Hamiltonian has been defined by the Legendre transformation in the multisymplectic formalism [23], and we have denoted the momentum by using the coordinate of Lagrangian systems for simplicity. The time variation in the Hamiltonian is given by 


$$
\begin{aligned}
\frac{\delta \mathcal{H}}{\delta t}=\int_{0}^{L}\left(A_{\rho}\right. & \left.w_{t} \frac{\delta w_{t}}{\delta t}+w_{t} \frac{\delta}{\delta t} \frac{\partial \mathcal{L}}{\partial w_{t}}-A_{\rho} w_{t} \frac{\delta w_{t}}{\delta t}+E I w_{x x} \frac{\delta w_{x x}}{\delta t}\right) d x \\
= & \int_{0}^{L}\left\{w_{t}\left(\frac{\partial}{\partial t} A_{\rho} w_{t}\right)+E I w_{x x}\left(\frac{\partial}{\partial t} w_{x x}\right)\right\} d x=-\int_{0}^{L}\left(e_{p} f_{p}+e_{q} f_{q}\right) d x
\end{aligned}
$$

where $\delta / \delta t$ is the variational derivative with respect to time, and the variational derivatives are regarded as partial derivatives.

\section{A.2. Calculation of Boundary Variables}

The second term of the integrand in the second equality of (A2) can be transformed:

$$
\begin{aligned}
\int_{0}^{L}\left(E I w_{x x} w_{x x t}\right) d x & =\int_{0}^{L}\left(-E I w_{x x x} w_{x t}\right) d x+\left[E I w_{x x} w_{x t}\right]_{x=0, L} \\
= & \int_{0}^{L}\left(E I w_{x x x x} w_{t}\right) d x+\left[E I w_{x x} w_{x t}-E I w_{x x x} w_{t}\right]_{x=0, L} \\
= & \int_{0}^{L}\left(E I w_{x x x x} w_{t}\right) d x+\left(e_{1}^{L} f_{1}^{L}+e_{2}^{L} f_{2}^{L}\right)-\left(e_{1}^{0} f_{1}^{0}+e_{2}^{0} f_{2}^{0}\right)
\end{aligned}
$$

where we have used the Stokes theorem to change the spatial integrals into boundary terms, and we have applied integration by parts to the first term of each equation. Substituting (A3) into (A2), we obtain the relation:

$$
\begin{gathered}
\frac{\delta \mathcal{H}}{\delta t}=\int_{0}^{L}\left(A_{\rho} w_{t t}+E I w_{x x x x}\right) w_{t} d x+\left(e_{1}^{L} f_{1}^{L}+e_{2}^{L} f_{2}^{L}\right)-\left(e_{1}^{0} f_{1}^{0}+e_{2}^{0} f_{2}^{0}\right) \\
=-\int_{0}^{L}\left(e_{p} f_{p}+e_{q} f_{q}\right) d x
\end{gathered}
$$

The integral term of the first equality in (A4) must vanish, because it is the system equation itself. Therefore, we obtain the power balance Equation (4) from $\delta \mathcal{H} / \delta t=0$.

(C) 2012 by the authors; licensee MDPI, Basel, Switzerland. This article is an open access article distributed under the terms and conditions of the Creative Commons Attribution license (http://creativecommons.org/licenses/by/3.0/). 